\title{
Response of different osmotic membranes and calibration of a coupled model capturing the temperature effect for the osmotic method
}

\author{
Shengyang Yuan ${ }^{1}$, Xianfeng Liu ${ }^{1, a}$, Olivier Buzzi ${ }^{1}$ \\ ${ }^{1}$ Priority Research Centre for Geotechnical and Materials Modelling, School of Engineering, The University of Newcastle, NSW, Australia.
}

\begin{abstract}
Reliable suction control is of paramount importance for experimental unsaturated soil mechanics. The osmotic method, based on polyethylene glycol (PEG) and semi-permeable membranes, is one of the possible methods to control suction. Although this method is quite simple to implement, reliable results can only be obtained with adequate calibration curves linking suction and PEG concentration, and a good understanding of the factors influencing the method. Several studies have investigated different aspects of this method, including the osmotic potential generated by given PEG concentrations, but the effect of temperature is not yet fully captured. This paper presents the results of a comprehensive study of the osmotic method looking at the long-term response of three different types of membranes, the effect of temperature, PEG molecular weight (MW) and the combination of different MW cut off (MWCO) on the calibration curves. Finally the data were used to calibrate a model capturing the temperature effect, in a coupled manner, in order to provide a robust calibration.
\end{abstract}

\section{Introduction}

The osmotic technique is one of the techniques available to control soil suction in experimental unsaturated soil mechanics [1-4]. The technique relies on an osmotic solution and semi-permeable membranes preventing the chemical species of the osmotic solution from diffusing into the soil tested. The suction generated within the soil specimen depends on the concentration of polyethylene glycol (PEG) and, in order to obtain reliable results, it is crucial to have a reliable and quality correlation between suction generated, PEG concentration, temperature and any other parameters that might influence the magnitude of suction. This correlation is referred to as a calibration curve.

Many authors have investigated the relationship between suction and PEG concentration but the effect of temperature has not been investigated comprehensively and is not accounted for explicitly in the calibration curves published in the literature. In the 1970s, Michel and Kaufmann showed that suction generated by PEG solutions decreases as temperature increases. This was recently confirmed by Tang and co-workers al. [5]. Such experimental observations are not in agreement with thermodynamics based models such as Van't hoff equation, which tend to predict an increase of suction as temperature increases. The data presented in this paper are part of a study aiming at ascertaining the quality of calibration of the osmotic technique and providing comprehensive dataset to capture and predict the effect of temperature on the magnitude of suction generated by the

\footnotetext{
a Corresponding author: Xianfeng.Liu@newcastle.edu.au
}

osmotic method. Three membrane types were tested, and their long-term response was studied. After deciding upon the most adequate membrane types, other tests were performed to establish the relationship between PEG concentration, PG molecular weight and temperature.

\section{Materials and Methods}

Four different types of PEG were used for this study (PEG 6000, 12000, 20000 and 35000). All were supplied by Sigma Aldrich, Germany. The PEG solutions were prepared by mixing the appropriate mass of PEG in 60 grams of deionized water. A magnetic stirrer was used to ensure good mixing. In terms of membranes, three types were used:

- Polythersulfon membranes (noted PES, supplied by Sartorius Stedim Biotech GmbH, Germany). Molecular weight cut off (MWCO) of 1000, 5000 and 10000 were used.

- Cellulose Acetate membrane (noted CA, supplied by Sigma Aldrich, Germany). MWCO of 8000.

- A ceramic membrane (noted CM, supplied by Sterlitech) with a single MWCO of 5000.

The set up used to measure the suction generated by the osmotic system in shown in Figure 1a. It involves a high capacity tensiometer [7] directly in contact with the semi permeable membrane. The whole set up was placed in a controlled temperature environment $\left( \pm 0.1^{\circ} \mathrm{C}\right)$. Unlike the PES and CA membranes that look like a supple plastic film, the ceramic membrane is made of a hard ceramic substratum with an active layer on one side 
(Figure 1c). The pore size distribution of the ceramic membrane shows a dominant pore size of about 5 microns (Figure 1b), which translates into an air entry in the order of $300 \mathrm{kPa}$. This implies that the active layer should be placed in contact with the soil specimen (or the tensiometer) and the ceramic part has to be immersed in the PEG solution. Otherwise, there is a risk of desaturation of the ceramic during testing. Moisture transfer would then occur via the vapour phase, which known to be a slow process.

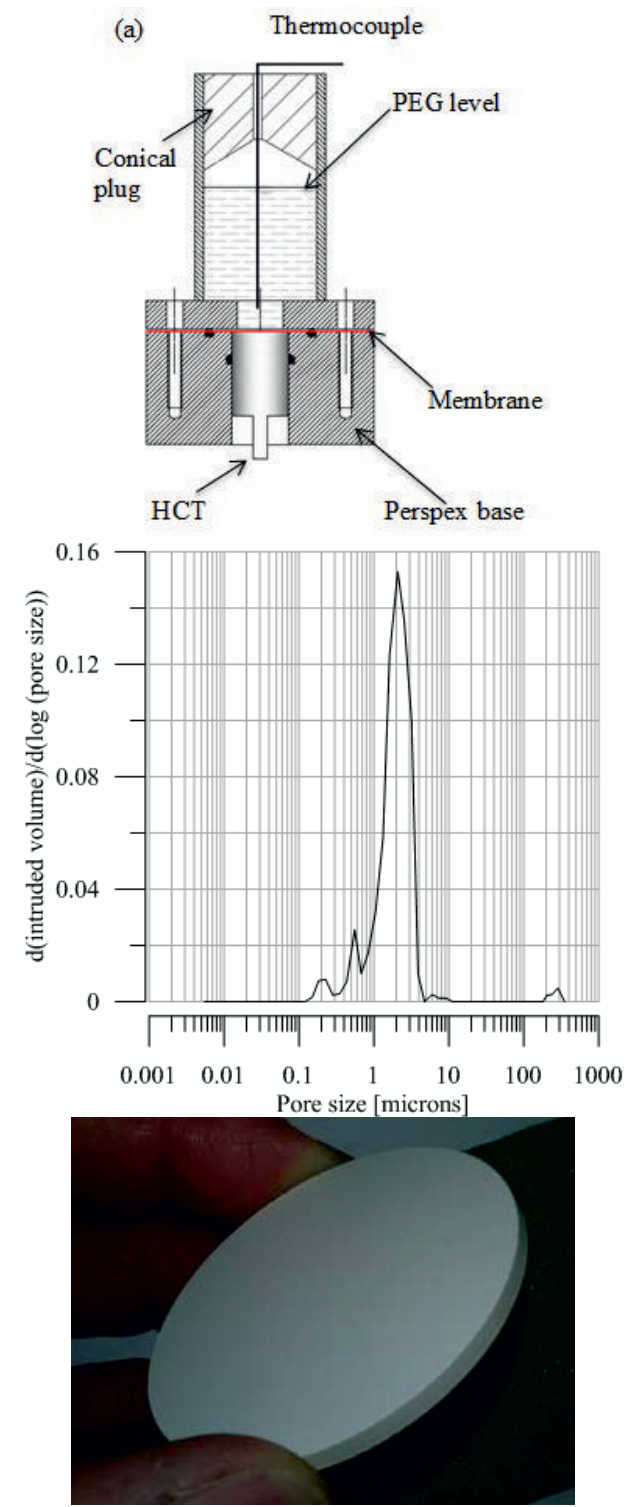

Figure 1. (a) Setup for the direct measurement of suction generated by the osmotic system. (b): Pore size distribution of the ceramic membrane obtained by mercury porosimetry. (c): View of the ceramic membrane and its active layer.

\section{Testing Program}

The test undertaken for this study are summarised below:

- Test series \#1 focuses on the general response of the CA, PES and CM membranes. Only one PEG concentration per membrane, one temperature (20 degrees), one PEG molecular weight (MW) and one molecular weight cut off (MWCO) were used.

- Test series \#2 investigates the suction generated by a combination of PES membranes, PEG molecular weight and different temperatures. For this part, four PEG MW (6000, 12000, 20000 and 35000), four PEG concentrations (about $0.15,0.20,0.25$ and $0.35 \mathrm{~g}$ $\mathrm{PEG} / \mathrm{g}$ water) and five temperatures $(20,25,30,35$ and 40 degrees) were selected.

\section{Results}

\subsection{Equilibration time and durability of membranes}

Figure 2 shows the response of the three membrane types for testing times in excess of 700 hours. Three different behaviours are clearly visible. Equilibration is reached very quickly for the CA membrane (about 4 to 5 hours), after which the suction measured by the tensiometer drops progressively and continuously until a stabilization at about 500 hours. Such response was observed for all tests with the CA membrane. This reflects an issue of stability of the membrane, a phenomenon pointed out by Tarantino and Mongiovi [3] and Tripathy and co-workers [8], among others.

The PES membrane requires more than 50 hours for the equilibration to be reached. Afterwards, the suction is sustainably maintained for about 24 hours. A slight increase in suction can be observed in Figure 2 from 70 hours onwards, which is the result of a slight evaporation of the PEG solution, yielding an increase in concentration and, hence, in suction. This was proved from a change in Brix index, from $20.3 \%$ before testing, to $21.9 \%$ at the end. This equates to a change of PEG concentration from 0.3 to $0.33 \mathrm{~g}$ PEG/g water. Regardless of the slight evaporation, the PES membrane remained functional for about 800 hours before the test was terminated.

As far as the authors are aware, there is no data in the literature on ceramic membranes. The preliminary results showed here suggested that the equilibration time is much longer than that of PES and CA membranes. Indeed, it takes more than 100 hours to reach equilibrium. Testing with this membrane showed no sign of degradation and this type of membranes might be more suited when long term testing is required (e.g. control of suction for soil testing with multiple cycles). 


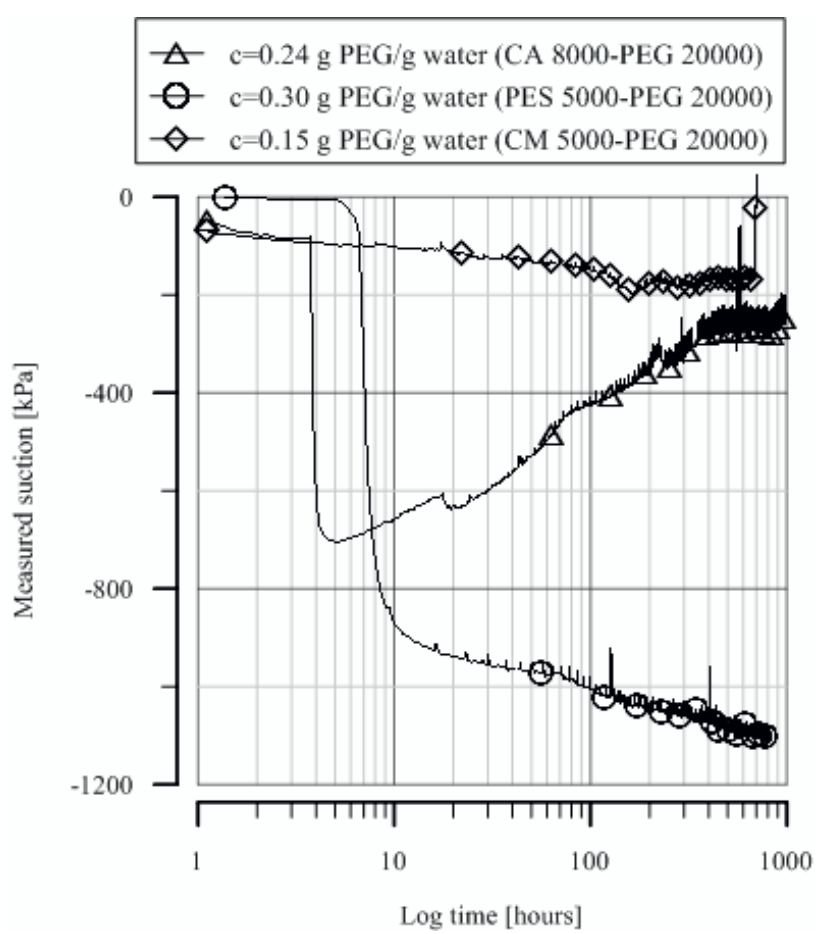

Figure 2. Evolution of suction with time for CA, PES and CM membranes.

At this stage, testing of the ceramic membrane is in progress and the rest of the paper will focus on new data pertaining to PES membranes which exhibit a relatively short equilibration time and good stability.

\subsection{Relationship between concentration, suction and temperature}

Figure 3 displays the data obtained at 20 degrees, for a range of PEG $\mathrm{MW}$ and two different membranes molecular weight cut off.

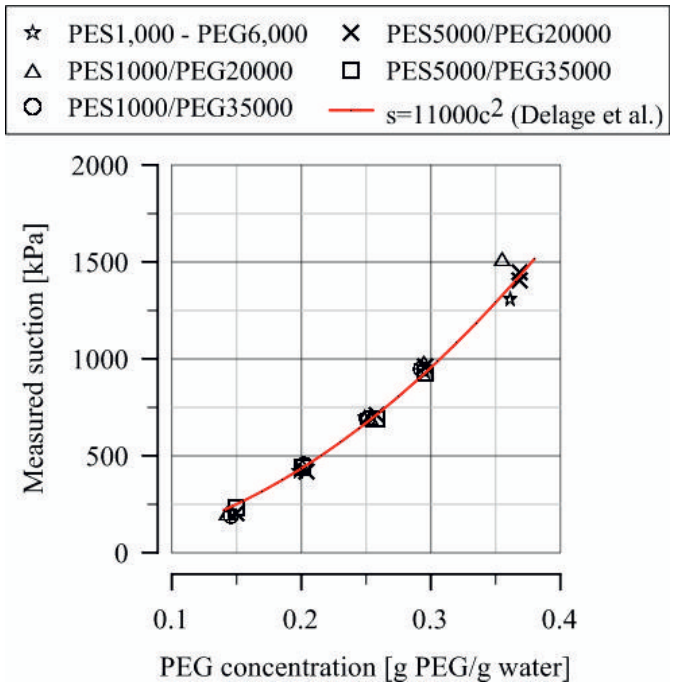

Figure 3. Values of measured suction against PEG concentration for different PEG MW and PES membranes MWCO.
All measured suctions are found to fall very close to the equation proposed by Delage and co-workers [4] that reads:

$$
s=11000 c^{2}
$$

with $c$ in $\mathrm{g} P E G / g$ water and $s$ in $\mathrm{kPa}$.

These data confirm that, regardless of the molecular weight and at 20 degrees, equation (1) applies to a number of combinations of PES membranes and PEG molecular weights, as long as no diffusion of PEG molecule occurs across the membrane. Such a result that was already observed by other researchers shows the robustness of equation (1) as a calibration equation.

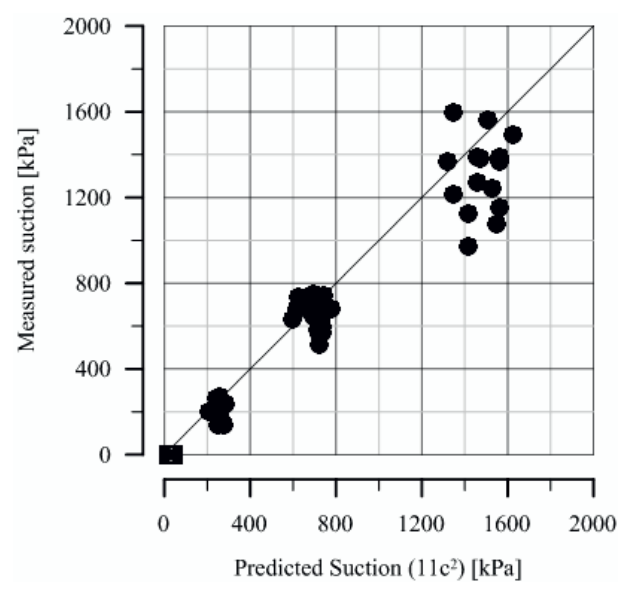

(a)

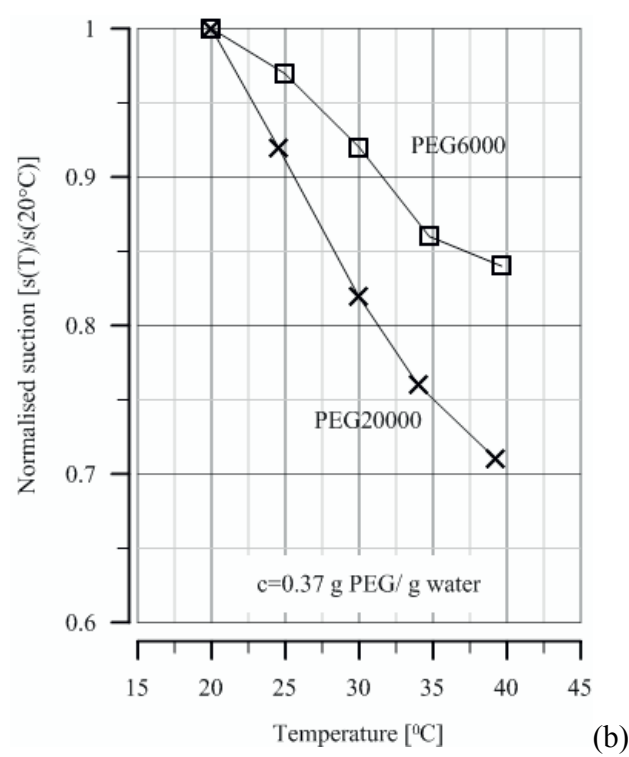

Figure 4. (a) Comparison of measured suction for different PEG MW, PEG concentration and temperature plotted against the predicted value, using Equation 1. (b): Reduction of suction (expressed in a normalised manner) with increasing temperature for PEG6000 and PEG20000. The normalised suction is the suction at temperature $\mathrm{T}$ over the suction at 20 degrees, which is about $1500 \mathrm{kPa}$.

Increasing the temperature produces an interesting outcome. Figure 4 plots the measured suction (at different temperatures) against the predicted suction (using Equation (1)). This time, a large scattering of data, more 
pronounced for high values of suction, is visible. In particular, the measured suction is systematically lower than its predicted counterpart. This trend is confirmed by Figure $4 \mathrm{~b}$ where the normalised suction drops as temperature increases. Interestingly, the magnitude of suction generated by the osmotic system is now dependent on the PEG molecular weight.

These experimental results are consistent with existing data of the literature $[5,6]$ but do not agree with thermodynamics based models, such as the virial model [9] or Van't Hoff equation, used to estimate osmotic pressure. Indeed, such models generally predict an increase of osmotic pressure as temperature rises:

$$
\pi / c \cong R T\left(A_{1}+A_{2} c+A_{3} c^{2}+\cdots\right)
$$

The present results suggest that a complete calibration of the osmotic method should account for the temperature, PEG molecular weight as well as PEG concentration.

\subsection{A coupled model to predict the suction generated by the osmotic system}

Michel and Kaufmann [6] proposed an empirical equation that captures the observed experimental results with terms coupling concentration and temperature:

$$
s=A_{1} c+A_{2} c^{2}+A_{3} c T+A_{4} c^{2} T
$$

where $s$ is the suction $[\mathrm{kPa}], c$ is the PEG concentration [g PEG/g water], $T$ is the temperature $\left[{ }^{0} \mathrm{C}\right], A_{1}, A_{2}, A_{3}, A_{4}$ are fitting coefficients.

For the PEG20000, the empirical model is calibrated as:

$$
S=-1500 \cdot c+21000 \cdot c^{2}+48 \cdot c T-365 \cdot c^{2} T
$$

Figure 5 shows the calibrated model (lines) against the experimental data points. The coupled equation adequately captures the experimental trends and can be considered an efficient tool to correct suction values in unsaturated soil testing should temperature fluctuate. Note that using a different PEG molecular weight would require re-calibrating the model.

\section{Conclusions}

This experimental study investigated the response of three types of semi-permeable membranes. Both CM and PES membranes seem durable but the equilibration time of the CM membrane is much longer than for the PES membrane. At this stage, testing on the ceramic membrane is to continue. The series of tests on the PES membranes conducted at 20 degrees Celsius show that the results are largely in agreement with the equation proposed by Delage and co-workers [4] and are independent of the PEG molecular weight and membrane molecular weight cut off (as long as no diffusion of PEG molecules occur across the membrane). However, it was found that the PEG molecular weight does influence the suction generated when the temperature is increased. A consistent reduction in suction was observed with a clear dependence on the PEG molecular weight. The experimental results were used to calibrate a coupled empirical model that can capture the evolution of suction generated as a function of PEG concentration and temperature hence providing a robust solution to adjust values of suction should the temperature fluctuate during testing.

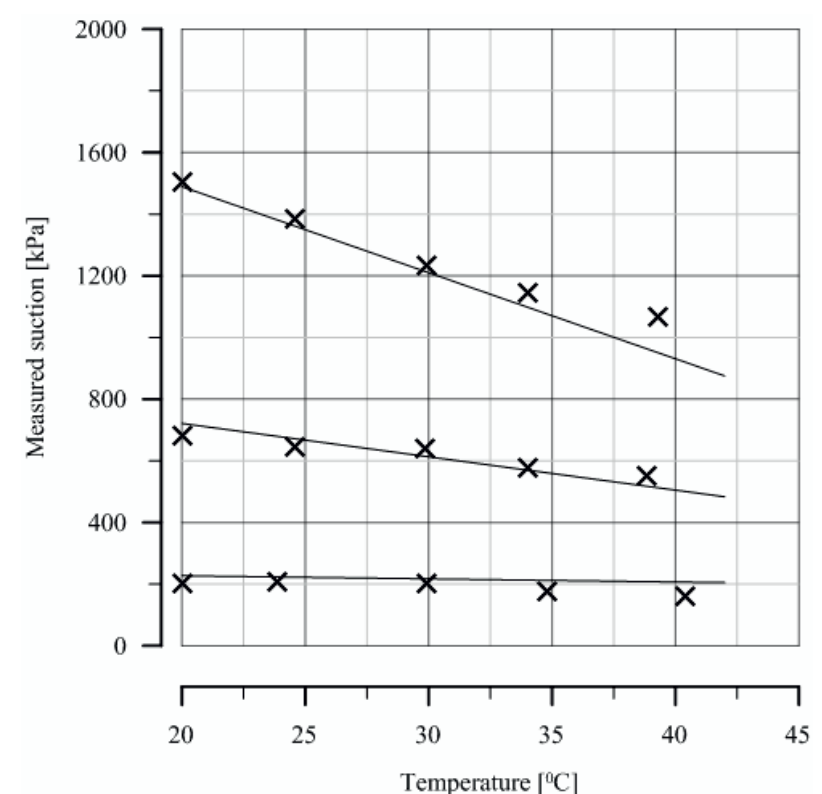

Figure 5. Calibrated model (Equation 4, for PEG20000) represented by the lines and experimental values of measured suction for different temperatures and PEG concentrations. Highest suction values correspond to 0.35 g PEG/g water, the middle ones to $0.25 \mathrm{~g} \mathrm{PEG/g}$ water and the lowest ones to $0.15 \mathrm{~g}$ PEG/g water.

\section{References}

1. G. Kassiff and A. Ben Shalom Géotechnique, 21, 245-255 (1971)

2. R. Monroy, A. Ridley, K. Dineen, K. and L. Zdravkovic ASTM Geotech. Test. J. 30, 1-7 (2007)

3. A. Tarantino, L. Mongiovi, In: Toll, D.G., Leong, E.C. (Eds.), Unsaturated Soils for Asia. Balkema, pp. 303-308 (2000)

4. P. Delage, M. Howat and Y.J. Cui. Eng. Geology, 50 (1-2) : 31-48 (1998).

5. A.M. Tang, Y.J. Cui, L.X. Qian, P. Delage and W.M. Ye. Can. Geotech. J. 47, 359-365, 2010.

6. B.E. Michel and M.R. Kaufmann, Plant Physiol. 51, 914-6. (1973)

7. J. Mendes and O. Buzzi. Can. G. J. 50, 550-556 (2013)

8. S. Tripathy, M.Y.M. Tadza and H.R. Thomas Géotechnique Lett. 1, 47-51. (2011)

9. P.J. Flory. Principles of polymer chemistry. Cornell University Press (1953). 